\title{
Análisis comparativo de modelos de madurez en inteligencia de negocio
}

\author{
Comparative analysis of maturity models in business intelligence
}

\author{
Roberto Prieto Morales $^{1} \quad$ Claudio Meneses Villegas ${ }^{1} \quad$ Vianca Vega Zepeda $^{1}$ \\ Recibido 9 de abril de 2014, aceptado 27 de octubre de 2014 \\ Received: April 9, 2014 Accepted: October 27, 2014
}

\begin{abstract}
RESUMEN
En el área de Business Intelligence (BI) se han planteado varios modelos de madurez, con distintos orígenes y propósitos. Dentro de esta diversidad, actualmente no se dispone de una comparación cuantitativa o cualitativa de estos modelos que entregue argumentos para seleccionar y utilizar uno de ellos como referencia en la mejora de la madurez en BI para una organización. En el presente artículo se aplica el método de estudio de similitudes y estándares (MESME) y la técnica de análisis envolvente de datos (DEA) para caracterizar y comparar un conjunto de modelos de madurez en BI previamente seleccionados. Con la aplicación del método MESME se identificaron y compararon las similitudes existentes entre los distintos modelos de madurez en BI. Por otro lado, con la implementación de la técnica DEA se obtuvo una caracterización de la capacidad cuantitativa de los diferentes modelos en una determinada fase para transformar las entradas en salidas, en los distintos niveles de madurez para un conjunto de modelos analizados. El resultado de esta investigación fue la obtención de una comparación cuantitativa y cualitativa de un conjunto de modelos de madurez de BI. Esto permitirá seleccionar uno de ellos como base para, por ejemplo, la elaboración de una guía metodológica enfocada a implementar mejoras en la madurez en BI para una organización.
\end{abstract}

Palabras clave: Inteligencia de negocio, inteligencia empresarial, modelos de madurez, comparación de modelos de madurez para BI.

\section{ABSTRACT}

Several maturity models have risen in the area of Business Intelligence (BI), with different origins and purposes. Within this diversity, there is currently no quantitative and / or qualitative comparison of them that give arguments to select and use one of them as reference in improving BI maturity for an organization. In this paper the method of study of similarities and standards (MESME) and the technique of data envelopment analysis (DEA) to characterize and compare a set of maturity models in selected BI applies. With the application of the method MESME identified and compared the similarities between the different models of BI maturity. On other side, with the implementation of the DEA technique, a quantitative characterization of the ability of different models in a given phase was obtained to transform inputs into outputs, at different levels of maturity for a set of models analyzed. The result of this research was to obtain a quantitative and qualitative comparison of a set of BI maturity models. This will allow selecting one of them as a basis for example for the development of a methodological guide focused on implementing improvements in BI maturity for an organization.

Keywords: Business intelligence, enterprise intelligence, maturity models, comparison of maturity models for $B I$.

\footnotetext{
1 Departamento de Ingeniería de Sistemas y Computación. Universidad Católica del Norte. Av. Angamos 0610. Antofagasta, Chile. E-mail: robertoprietomorales@gmail.com; cmeneses@ucn.cl; vvega@ucn.cl
} 


\section{INTRODUCCIÓN}

Actualmente las organizaciones deben diferenciarse de su competencia tomando mejores decisiones de negocios en el menor tiempo posible en comparación con las otras organizaciones. Para ello las organizaciones deben contar con la mayor cantidad de información disponible que apoye el proceso de toma decisiones de negocio.

En este contexto se presenta BI como un conjunto de herramientas y soluciones tecnológicas diseñadas para que los usuarios puedan extraer de manera eficiente información empresarial útil para apoyar el proceso de toma de decisiones, obteniendo una ventaja competitiva sobre otras organizaciones [1].

Según Wixom y Watson [2], BI es "una amplia categoría de tecnologías, aplicaciones y procesos para la recolección, almacenamiento, acceso y análisis de los datos, para ayudar a sus usuarios a tomar mejores decisiones". Para Jourdan [3] "BI es el proceso que analiza la información que reside en la empresa, a fin de mejorar su proceso de decisión, y por consiguiente crear una ventaja competitiva para la empresa".

El origen en BI fue en primera instancia introducido por Gartner Group [4], refiriéndose incipientemente a algunas herramientas y tecnologías que incluían almacenes de datos, informes y análisis de consultas. En la actualidad BI es considerado como una solución de gran alcance, una herramienta extremadamente valiosa y clave para adicionar valor a la organización.

Las organizaciones para poder implementar iniciativas de BI deben realizar una inversión económica considerable. Por lo que buscan rentabilizar lo mayor posible su retorno de inversión ROI (Return On Investment). Además las organizaciones necesitan medir cómo se encuentran respecto de sus iniciativas de BI, para poder compararse con su competencia.

Rajteric [5] sugiere los modelos de madurez para enunciar una correcta línea base, para la medición de valor en sus iniciativas en BI, además de entender dónde están y cómo pueden mejorar.

El origen del concepto de modelo de capacidad de madurez (Capability Maturity Model, CMM) [6] se origina en el Software Engineering Institute
(SEI), Carnegie Mellon University en USA. Este fue propuesto por W. Humphrey en 1986, proporcionando un modelo de capacidad de madurez enfocado a las mejores prácticas para el proceso de desarrollo de software.

Los modelos de madurez en BI posibilitan a las organizaciones medir y comparar sus iniciativas en ese ámbito. Además, indican el estado actual de madurez en el que se encuentra la organización y describen el camino hacia dónde debe moverse desde su ubicación actual.

Existen varios modelos de madurez en BI, algunos fueron creados por la academia y otros por organizaciones privadas que se dedican a comercializar productos de BI. Estos modelos de madurez en BI poseen enfoques diversos tales como sistemas, procesos y tecnologías, entre otros.

En este artículo se realiza una caracterización cuantitativa de los distintos niveles de madurez en un conjunto de modelos de madurez en BI que son objeto de análisis. Para ello se aplicó la técnica DEA [7], con donde se logró describir la eficiencia en los diferentes niveles de madurez para cada uno de los modelos de madurez en BI analizados.

Además, se desarrolla una comparación cualitativa entre los modelos de madurez en BI, aplicando el método MESME [8]. Con el uso de este método fue posible analizar las similitudes presentes, para ello se estableció un marco de referencia con las directrices para comparar los modelos de madurez en BI seleccionados.

La presente investigación tiene como objetivo realizar una comparación para seleccionar un modelo de madurez en BI, para tomarlo como referencia en futuros trabajos que implementen mejoras en la madurez en BI para una organización. Existen otras revisiones o comparaciones de modelos de madurez en BI [5, 9-10], ninguna de estas comparaciones realiza una asimilación cualitativa y cuantitativa de los modelos de madurez en BI a la vez.

El resto del artículo está estructurado como se indica a continuación. La sección denominada Materiales y Métodos contempla el desarrollo de una caracterización de los conceptos de los modelos de madurez, una revisión de los modelos 
de madurez en BI que serán objetos de análisis, y una definición general de la técnica DEA. La sección denominada Eficiencia de un Modelo de BI describe una adaptación de la técnica DEA para medir cuantitativamente un aspecto de la eficiencia de un modelo de BI. La sección denominada Similitud entre Modelos de BI considera la aplicación del método MESME para establecer un cuadro comparativo en términos de similitudes de los distintos modelos de BI considerados. En la sección Resultados se enuncian las razones de la elección de uno de los modelos como modelo de referencia para efectos de establecer una guía de mejoramiento BI. Finalmente, se enuncian las conclusiones obtenidas de este trabajo.

\section{MATERIALES Y MÉTODOS}

\section{Modelos de madurez en BI}

Los modelos de madurez en BI se usan para describir, explicar y evaluar los ciclos de crecimiento de vida en iniciativas de BI. El concepto básico de la mayoría de los modelos de madurez en BI es que las cosas cambian en el tiempo, y gran parte de esos cambios se pueden regularizar.

Los modelos de madurez deben tener ciertas características como el concepto de madurez, dimensiones, niveles, principio de madurez y un enfoque de evaluación [10]. A continuación se describen las características de los modelos de madurez en BI.

a) Concepto de Madurez: Es determinado por factores como la capacidad de las personas, la madurez de los procesos y la madurez de la tecnología.

b) Dimensiones: Son áreas de capacidad específicas, o áreas de proceso que estructuran un campo de interés.

c) Nivel: Corresponde a los estados del modelo de madurez para un cierto dominio.

d) KPA: Área de Proceso Clave.

e) Principio de madurez: Los modelos de madurez pueden ser continuos o por niveles.

f) Evaluación: El enfoque de evaluación puede ser cualitativo o cuantitativo.
La Tabla 1 enuncia las características de un conjunto de modelos de madurez en BI seleccionados previamente. Este conjunto de modelos de madurez en BI se obtuvo como el resultado de una búsqueda realizada entre las principales fuentes digitales del conocimiento, seleccionando los modelos de madurez en BI más importantes, según la definición de expertos.

Tabla 1. Conjunto de Modelos de Madurez en BI

\begin{tabular}{|c|c|}
\hline Modelo & Descripción \\
\hline $\begin{array}{l}\text { Modelo de Capa- } \\
\text { cidad de Madurez } \\
\text { de Enterprise } \\
\text { Intelligence (EI) } \\
\text { [11] (J. Huffman } \\
\text { y L. Whitman) }\end{array}$ & $\begin{array}{l}\text { El concepto de Enterprise Intelligen- } \\
\text { ce (EI) es más amplio que BI, y abar- } \\
\text { ca no solo el análisis de los datos, } \\
\text { sistemas y procesos, sino también } \\
\text { la arquitectura y la gestión del cono- } \\
\text { cimiento. EI posee cinco niveles de } \\
\text { madurez y tres dimensiones: datos, } \\
\text { procesos y sistema. }\end{array}$ \\
\hline $\begin{array}{l}\text { Modelo Enter- } \\
\text { prise Business } \\
\text { Intelligence Ma- } \\
\text { turity (EBIM) } \\
\text { [12] (Tan, Sim y } \\
\text { Yeoh) }\end{array}$ & $\begin{array}{l}\text { Este modelo se emplea para la ges- } \\
\text { tión de una empresa respecto de sus } \\
\text { iniciativas de inteligencia empresa- } \\
\text { rial, posee cinco niveles de madurez, } \\
\text { en los cuales describe la evolución } \\
\text { de la madurez para sus cuatro dimen- } \\
\text { siones. }\end{array}$ \\
\hline $\begin{array}{l}\text { Modelo de Ma- } \\
\text { durez Service } \\
\text { Oriented Busi- } \\
\text { ness Intelligence } \\
\text { (SOBI)[13] } \\
\text { (Shaaban, Helmy, } \\
\text { Khedr y Nasr) }\end{array}$ & $\begin{array}{l}\text { El modelo SOBI combina dos para- } \\
\text { digmas arquitectónicos que se desa- } \\
\text { rrollan en forma independiente, estos } \\
\text { son BI y servicios. Este modelo de } \\
\text { madurez posee cinco niveles, para } \\
\text { las dimensiones tecnología, organi- } \\
\text { zación y experiencia en negocios. }\end{array}$ \\
\hline $\begin{array}{l}\text { Modelo The Data } \\
\text { Warehousing } \\
\text { Institute (TWDI) } \\
\text { [14] (Wayne } \\
\text { Eckerson) }\end{array}$ & $\begin{array}{l}\text { El modelo TDWI se divide en cinco } \\
\text { niveles desde la infancia hasta la sa- } \\
\text { biduría. Para la elaboración de este } \\
\text { modelo se tomó como referencia el } \\
\text { modelo CMMI. }\end{array}$ \\
\hline $\begin{array}{l}\text { Modelo de Ma- } \\
\text { durez Enterprise } \\
\text { Business Intelli- } \\
\text { gence (EBI2M) } \\
\text { [15] } \\
\text { (Chuah y Wong) }\end{array}$ & $\begin{array}{l}\text { El modelo EBI2M posee una repre- } \\
\text { sentación continua, compuesta por } \\
\text { trece dimensiones para sus cinco ni- } \\
\text { veles de madurez. }\end{array}$ \\
\hline
\end{tabular}

\section{Data Envelopment Analysis (DEA)}

DEA es una técnica de optimización desarrollada por Charnes, Cooper y Rhodes [7], elaborada para medir el comportamiento relativo de distintas unidades de toma de decisión (Decision Making Unit, DMU). 


\section{EFICIENCIA DE UN MODELO DE BI}

La presente sección describe una adaptación de la técnica DEA aplicado a determinar la eficiencia en centros de producción [16]. Esta adaptación de la técnica DEA considera entender la eficiencia como la capacidad cuantitativa de cada modelo en una determinada fase para transformar las entradas en salidas en cada uno de los niveles de madurez aplicados al conjunto de modelos descritos en la Tabla 1. La aplicación de la técnica DEA implica la contabilización de los elementos de entrada y salida presentes en cada uno de los niveles de madurez de los modelos. Esto posibilita comparar la composición estructural presente en cada nivel de madurez entre los modelos en consideración. A partir de esto se calcula la eficiencia, la que posibilita reconocer los cambios en la relación entre los elementos de entrada y salida considerando todos los niveles de madurez. Con esto, la eficiencia permite identificar los modelos que presentan una estructura rígida en sus niveles de madurez, y los modelos que poseen una estructura flexible que varía según las dificultades propias de cada nivel de madurez. La Figura 1 resume las etapas de la aplicación de la técnica DEA.

\begin{tabular}{|l|l|}
\hline $\begin{array}{l}\text { A. Definición y } \\
\text { selección de las } \\
\text { DMU }\end{array}$ & $\begin{array}{l}\text { F. Presentar los } \\
\text { resultados }\end{array}$ \\
\hline \multicolumn{1}{|c|}{$\begin{array}{l}\text { B. Definición } \\
\text { selección y } \\
\text { medición de input } \\
\text { y output }\end{array}$} \\
\begin{tabular}{|l|l|}
\hline \multicolumn{1}{|c|}{$\downarrow$} \\
\begin{tabular}{|l|} 
C. Selección y \\
formulación del \\
modelo DEA
\end{tabular} \\
\hline
\end{tabular} \\
\hline
\end{tabular}

Figura 1. Etapas de técnica DEA.

\section{A. Definición y Selección de las DMU}

La DMU será cada uno de los niveles de madurez a analizar. Como la mayoría de los modelos de madurez en BI poseen una estructura de cinco niveles de madurez, se seleccionarán todos los modelos de madurez que cumplan con dicho criterio.
La Figura 2 muestra la correspondencia de cada una de las DMU con su respectivo nivel.

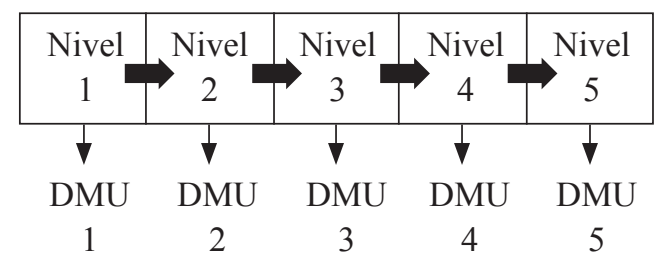

Figura 2. Selección de DMU.

\section{B. Definición, Selección y Medición de las Variables Input-Ouput}

En esta fase de la aplicación de la técnica DEA se establecen los criterios para identificar los input y output necesarios para establecer las eficiencias de las distintas DMU.

Los criterios para identificar los input son los siguientes:

- Cada una de las actividades que sean parte de la caracterización de un nivel en particular.

- Cada una de las herramientas de BI que se encuentren descritas como parte de un nivel en particular.

- Cada una de las metodologías de BI correspondientes a los elementos de un nivel en particular.

- Para el caso que el modelo tenga definidas las prácticas, solo se considerarán estas como input de la DMU.

- Para el caso que el modelo no posea una definición de práctica explícita, se considerará una práctica implícita para cada KPA que posea un nivel en particular.

Los criterios para los output son los siguientes:

- Cada una de las áreas de proceso clave KPA pertenecientes a un nivel en particular.

- En el caso de que el modelo no presente KPA, se considerará como un output a cada una de las dimensiones que posea un nivel en particular.

- Para el caso que el modelo no presente KPA ni dimensiones, se tomará como output el objetivo de un nivel o estado en particular.

Con lo que se cuantifican los input y los output en los distintos niveles de madurez para el conjunto de modelos a analizar, para ello se contabiliza 
cada criterio establecido explícitamente en la caracterización de cada modelo de madurez en BI seleccionados, tanto para los input como para los output.

\section{Selección y formulación de la técnica DEA}

En esta sección se describe un modelo matemático correspondiente a la aplicación empírica de la técnica DEA para la medición de la eficiencia en cada DMU o nivel.

La técnica DEA proporciona un método para comparar cuantitativamente la eficiencia de unidades organizacionales respecto de las demás. Como cada unidad organizacional está en un contexto distinto a las demás, existe una dificultad al establecer cuáles van a ser los criterios a utilizar para comparar la eficiencia de una unidad respecto de la otra. Por lo que es posible establecer una medida para cada unidad, expresada de la forma indicada en la Ecuación (1):

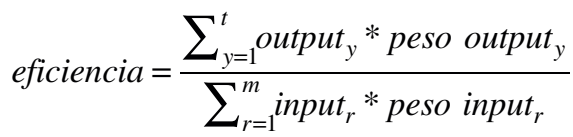

En donde $t$ es el número que determina la medida de eficiencia y $m$ es el número de productos. Para medir la eficiencia relativa de $n$ unidades se elaboran n modelos de optimización. En cada uno de estos modelos la función a maximizar es la eficiencia de la unidad organizacional $j(j=1,2, \ldots \mathrm{n})$.

\section{Aplicación de la técnica DEA}

En la presente fase de la técnica DEA se aplica la formulación descrita en la Ecuación (1). Por lo que primero se calcula la eficiencia teniendo en cuenta los input y los output para cada uno de los modelos de madurez.

En este caso se realizará la descripción completa del cálculo de la capacidad cuantitativa que presentan cada uno de los modelos en el nivel 1 o DMU 1 para transformar los input en output.

La Tabla 2 en las columnas inputs y outputs enuncia la cuantificación de los input y output para cada uno de los modelos de madurez en BI en el nivel 1. La columna KPA / Práctica muestra el cociente resultante entre estas variables. La última columna
Eficiencia relativa describe cada una de las unidades de decisión empleadas en la ecuación (1) para cada unidad de decisión perteneciente al nivel 1 .

La Tabla 2 muestra que las unidades de decisión EBIM, TDWI y EBI2M poseen los cocientes más altos $(1,0)$. En cambio, la unidad de decisión EI presenta el cociente más bajo $(0,266)$. Por tanto los modelos EBIM, TDWI y EBI2M realizan menos esfuerzos en generar sus salidas, en comparación al modelo EI que realiza un mayor esfuerzo en generar sus salidas.

Por lo anterior, las unidades de decisión EI y SOBI presentan una eficiencia menor que las unidades de decisión EBIM, TDWI y EBI2M, las cuales obtienen el máximo de eficiencia.

Tabla 2. Eficiencia nivel 1.

\begin{tabular}{|c|c|c|c|c|}
\hline \multirow{2}{*}{ 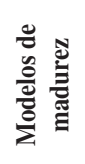 } & $\begin{array}{c}\mathrm{N}^{\circ} \\
\text { prácticas }\end{array}$ & $\begin{array}{c}\mathbf{N}^{\circ} \\
\text { KPA }\end{array}$ & \multirow{2}{*}{$\begin{array}{c}\mathbf{N}^{\circ} \mathbf{K P A} / \\
\mathrm{N}^{\circ} \\
\text { prácticas }\end{array}$} & \multirow{2}{*}{$\begin{array}{c}\text { Eficiencia } \\
\text { relativa }(\%)\end{array}$} \\
\hline & Input & $\begin{array}{l}\text { Out- } \\
\text { put }\end{array}$ & & \\
\hline EI & 15 & 4 & 0,266 & $\begin{array}{c}(0,266 / 1) * \\
100=26,6 \%\end{array}$ \\
\hline EBIM & 4 & 4 & 1 & $\begin{array}{c}(1 / 1) * 100 \\
=100 \%\end{array}$ \\
\hline SOBI & 9 & 3 & 0,333 & $\begin{array}{c}(0,333 / 1) * \\
100=33,3 \%\end{array}$ \\
\hline TDWI & 1 & 1 & 1 & $\begin{array}{c}(1 / 1) * 100 \\
=100 \%\end{array}$ \\
\hline EBI2M & 1 & 1 & 1 & $\begin{array}{c}(1 / 1) * 100 \\
=100 \%\end{array}$ \\
\hline
\end{tabular}

\section{E. Análisis envolvente de procedimientos}

En esta fase se desea poner una meta a los modelos de madurez para mejorar su eficiencia. Para ello se mantienen los inputs y se aumentan los outputs, esto se llama meta de outputs. Por lo que según la definición de expertos, se aumentan los outputs en $10 \%$, para ver la eficiencia de los modelos con la misma cantidad de inputs.

La Tabla 3 describe la incorporación de una nueva columna correspondiente a $10 \%$ adicional de la cantidad de output original. Esta columna se denomina salidas varias.

Para medir la eficiencia usando estos nuevos datos se divide cada nuevo output por cada input. La Tabla 4 describe los resultados obtenidos. 
Tabla 3. Salidas múltiples.

\begin{tabular}{|l|c|c|c|}
\hline \multirow{2}{*}{$\begin{array}{c}\text { Modelos de } \\
\text { madurez }\end{array}$} & Prácticas & KPA & $\begin{array}{c}\text { Salidas } \\
\text { Varias }\end{array}$ \\
\cline { 2 - 4 } & Input & Output & Output \\
\hline EI & 15 & 4 & 4.4 \\
\hline EBIM & 4 & 4 & 4.4 \\
\hline SOBI & 9 & 3 & 3.3 \\
\hline TDWI & 1 & 1 & 1.1 \\
\hline EBI2M & 1 & 1 & 1.1 \\
\hline
\end{tabular}

Tabla 4. Eficiencias particulares.

\begin{tabular}{|l|c|c|}
\hline Modelos de madurez & $\begin{array}{c}\text { KPA / } \\
\text { prácticas }\end{array}$ & $\begin{array}{c}\text { Salidas varias / } \\
\text { prácticas }\end{array}$ \\
\hline EI & 0,266 & 0,293 \\
\hline EBIM & 1 & 1,1 \\
\hline SOBI & 0,333 & 0,366 \\
\hline TDWI & 1 & 1,1 \\
\hline EBI2M & 1 & 1,1 \\
\hline
\end{tabular}

Se observa que los cocientes KPA / prácticas y Salidas varias / prácticas de las unidades de decisión EBIM, TDWI y EBI2M obtuvieron los más altos valores, es decir, máxima eficiencia.

\section{F. Presentación y análisis de resultados}

Con los resultados anteriores se aprecia lo dificultoso que es comparar unidades de decisión para determinar su eficiencia relativa. Por ejemplo, teniendo en cuenta los resultados de la Tabla 4 se observa desde la columna KPA / prácticas que EBI2M es 0,266 veces más eficiente que EI.

La Figura 3 muestra un gráfico con los resultados de la Tabla 4. En este gráfico se traza una línea horizontal desde los puntos EBIM, TDWI y EBI2M hasta el eje vertical, y se traza una línea vertical desde los mismos puntos hasta el eje horizontal. Estas dos líneas corresponden a la Frontera de Eficiencia.

La Frontera de Eficiencia se obtiene de las unidades de decisión que poseen la mejor manera de realizar sus procesos. Esta Frontera determina un estándar para las unidades de decisión que no están en dicha Frontera, constituyendo una meta a alcanzar.

\section{SIMILITUDES ENTRE MODELOS DE BI}

Este método fue propuesto por el grupo de investigación de la Universidad Politécnica de Madrid (MPSEI), siendo validado por los autores en diferentes ámbitos de estudio [8].

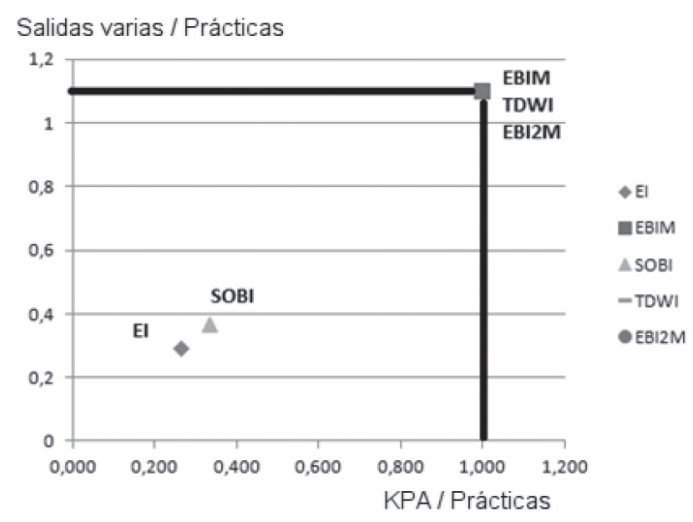

Figura 3. Frontera de eficiencia nivel 1.

El objetivo de la aplicación del método es que la comparación de modelos sea realizada bajo un análisis formal y debe estar soportada por un método claro y definido.

En esta sección se describe la adaptación del Método de Estudio de Similitudes y Estándares, para identificar similitudes entre un conjunto de modelos de madurez en BI. La Figura 4 describe las etapas que componen el método MESME.

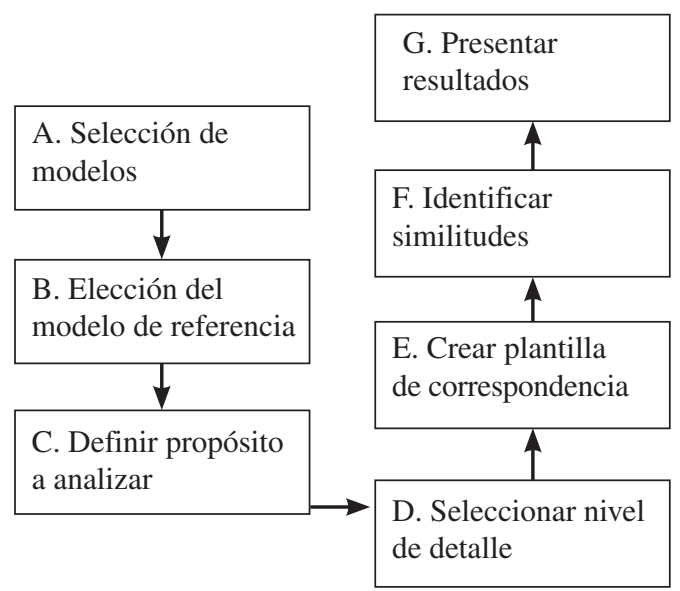

Figura 4. Etapas del método MESME.

\section{A. Selección de modelos}

Los modelos o estándares seleccionados, para ser analizados en la presente sección, son los modelos descritos en la Tabla 1.

\section{B. Elección del modelo de referencia}

Los autores han ponderado cada uno de los modelos de madurez en BI seleccionados, teniendo 
en consideración la presencia o ausencia de los elementos que conforman un modelo de madurez en BI, entre los cuales se encuentran los niveles, KPA, objetivos, prácticas y dimensiones.

La Tabla 5 muestra el resultado de la ponderación de cada uno de los modelos. Para ello se cuantificó cada criterio encontrado en cada modelo, para posteriormente promediar los totales obtenidos para cada modelo descrito en la ecuación (2).

$$
v=\frac{\sum_{i}^{n} W_{i}}{n}
$$

En donde $W$ es la cuantificación del criterio presente en cada modelo, $i$ es el porcentaje de ponderación asignado a cada criterio, y $V$ es el total obtenido de la Tabla 5.

Tabla 5. Ponderación de modelos.

\begin{tabular}{|l|c|c|c|c|c|}
\hline \multicolumn{1}{|c|}{ Modelos } & EI & EBI2 & TDWI & $\begin{array}{c}\text { SO } \\
\text { BI }\end{array}$ & EBI \\
\hline $\begin{array}{l}\text { Dimensios } \\
(20 \%)\end{array}$ & 4,0 & 5,0 & 4,0 & 4,0 & 4,0 \\
\hline Niveles (20\%) & 5,0 & 5,0 & 5,0 & 5,0 & 5,0 \\
\hline KPA (20\%) & 5,0 & 5,0 & 5,0 & 5,0 & 5,0 \\
\hline $\begin{array}{l}\text { Objetivos } \\
(20 \%)\end{array}$ & 5,0 & 2,0 & 5,0 & 2,0 & 5,0 \\
\hline $\begin{array}{l}\text { Prácticas } \\
(20 \%)\end{array}$ & 5,0 & 1,0 & 1,0 & 1,0 & 1,0 \\
\hline Total & 4,8 & 3,6 & 4,0 & 3,4 & 4,0 \\
\hline
\end{tabular}

La definición del criterio para la selección del modelo de referencia es a partir de los resultados de la ponderación de estándares definido en la sección anterior. Por lo que se recomienda que el modelo que mayor ponderación obtuvo sea el modelo de referencia. La elección del modelo de referencia no está relacionada con la aplicación de la técnica DEA implementada en la subsección Data Envelopment Analysis.

Según los resultados obtenidos en la Tabla 5, el modelo de referencia seleccionado es el modelo de madurez en BI Enterprise Intelligence, EI. Dicho modelo posee una completa caracterización en todos sus elementos como dimensiones, niveles, KPA, objetivos y prácticas.

Según Wells [17], "La inteligencia empresarial (Enterprise Intelligence, EI) es la capacidad de una organización o empresa de razonar, planear, predecir, resolver problemas, pensar de manera abstracta, comprender, innovar, aprender de manera que aumente los conocimientos de una organización, informar a los procesos de decisión, llevar a cabo acciones efectivas y ayudar a establecer y lograr los objetivos del negocio".

\section{Definir propósito a analizar}

El objetivo de la aplicación del método MESME es identificar las similitudes existentes en cada uno de los modelos de madurez en BI seleccionados, respecto del proceso elegido.

Con esto se busca complementar el proceso elegido con actividades definidas en los otros modelos de madurez en BI distintos al modelo de referencia.

El proceso seleccionado es la identificación de fuentes correspondientes al nivel 2 del modelo de madurez en BI Enterprise Intelligence. Este proceso se refiere a definir actividades que ayuden al manejo de contenidos, medios y tecnologías.

La Tabla 6 enuncia un resumen de los objetivos específicos y las prácticas específicas del proceso de identificación de fuentes.

\section{Seleccionar el nivel de detalle}

El nivel de detalle es un factor importante que permite destacar las correspondencias identificadas en los modelos o estándares respecto del modelo de referencia. Teniendo en consideración que en el método MESME se analizó la información de los términos comunes entre los distintos modelos y estándares seleccionados, los autores determinaron los siguientes:

- Analizar la estructura del modelo de referencia EI.

- Respetar la estructura del modelo de referencia EI.

- El nivel de prácticas específicas de los modelos de BI.

- El nivel de áreas de proceso clave se presenta en gran parte de los modelos seleccionados.

Teniendo en cuenta estas consideraciones, se concluye que las áreas de proceso clave será el nivel de detalle con que se analizarán los modelos seleccionados. Por lo que se busca identificar áreas 
Tabla 6. Objetivos y prácticas.

\begin{tabular}{|l|l|}
\hline Objetivo específico & Prácticas específicas \\
\hline $\begin{array}{l}\text { SG1 La capacidad de múltiples medios } \\
\text { de comunicación electrónicos en todas } \\
\text { las prácticas de producción y los docu- } \\
\text { mentos autorizados. }\end{array}$ & $\begin{array}{l}\text { SP1.1 Establecer las normas, prácticas y herramientas para varios tipos de } \\
\text { medios. } \\
\text { SP1.2 Ingeniero de medios de comunicación, contenido basado en el diseño y } \\
\text { construcción de registro. } \\
\text { SP1.3 Utilizar los contenidos multimedia para todas las comunicaciones empre- } \\
\text { sariales y productos de aprendizaje. }\end{array}$ \\
\hline $\begin{array}{l}\text { SG2 Administrar el contenido que com- } \\
\text { prende documentos de las empresas, pá- } \\
\text { ginas web, bases de datos, bases de cono- } \\
\text { cimiento, y otras fuentes de datos, cono- } \\
\text { cimiento del proceso, el conocimiento del } \\
\text { sistema, y el servicio. }\end{array}$ & $\begin{array}{l}\text { SP2.1 Determinar las clasificaciones de contenido. } \\
\text { SPrtenidos, el acceso y la ingeniería. } \\
\text { SP2.4 Desarrollar el contenido del medio ambiente orientado a los controles (de } \\
\text { escritorio, navegadores, portales, mash-ups, paneles de control, pizarras, etc). }\end{array}$ \\
\hline $\begin{array}{l}\text { SG3 Administrar el crecimiento de la red } \\
\text { y el cambio técnico. }\end{array}$ & $\begin{array}{l}\text { SP3.1 Desarrollar un plan para los cambios tecnológicos de la red. } \\
\text { SP3.2 Determinar el uso óptimo interno en comparación con los recursos de red } \\
\text { externos. } \\
\text { SP3.3 Colaborar con otras áreas clave del proceso para determinar las previsio- } \\
\text { nes de carga y prepararse para un aumento de la demanda. }\end{array}$ \\
\hline
\end{tabular}

de proceso claves que tengan objetivos similares a los objetivos del proceso seleccionado.

\section{E. Crear plantilla de correspondencia}

Continuando con las sugerencias del método MESME, se estableció una plantilla de correspondencia que permitiera establecer la similitud. La Tabla 7 muestra la validación de preguntas que establece la plantilla de correspondencia.

Tabla 7. Plantilla de correspondencia.

\begin{tabular}{|c|c|}
\hline Preguntas & Respuestas \\
\hline $\begin{array}{l}\text { ¿Existen áreas de proceso clave o } \\
\text { temas en el modelo EBI2M que se } \\
\text { relacione con el modelo de referen- } \\
\text { cia? }\end{array}$ & Sí \\
\hline $\begin{array}{l}\text { ¿Cuáles son las áreas de proceso } \\
\text { clave o temas que tiene el modelo } \\
\text { EBI2M que complementan el mo- } \\
\text { delo EI? }\end{array}$ & $\begin{array}{l}\text { - Cambio. } \\
\text { - Administración. }\end{array}$ \\
\hline
\end{tabular}

\section{F. Identificar similitudes}

A continuación se muestra el término del análisis de la subsección anterior, esta pretende determinar características de los modelos respecto del proceso de identificación de contenido, medios y tecnologías. Por tanto se comparan los modelos y se presentan los resultados en la Tabla 8 .

\section{G. Presentar resultados}

La Tabla 9 presenta los resultados correspondientes a la identificación de similitudes de temas de BI.

Se muestra solo una similitud en los temas Data Marts, Data Warehouse y Enterprise Data Warehouse para los modelos TDWI y EBI. Por lo que el modelo TDWI es el único que presenta similitudes con otros modelos. En cambio, los modelos EI, EBI2 y SOBI no presentan similitudes de temas con otros modelos.

Con esto se hace evidente la escasa similitud de temas entre los modelos, ya que la mayoría de ellos presentan una orientación específica, con la excepción de los temas relacionados a los almacenes de datos y los indicadores clave de desempeño, elementos esenciales en una arquitectura de BI.

\section{RESULTADOS}

La Tabla 10 muestra la cuantificación de la similitud de temas encontrados en el conjunto de modelos. Solo el modelo TDWI presenta coincidencias de temas con otros modelos, encontrándose una similitud de 3 temas con el modelo EBIM. Con esto se reafirma la exigua compenetración de temas que existe entre el conjunto de modelos de madurez en BI, ya que la mayoría presentan propósitos diversos.

La Figura 5 muestra un gráfico que resume la eficiencia de todos los modelos de madurez en BI. 
Tabla 8. Identificación de similitudes método MESME.

\begin{tabular}{|c|c|c|c|}
\hline Modelo & Nivel & Objetivo & KPA \\
\hline EI & 2 & $\begin{array}{l}\text { Se refiere a definir actividades que ayuden al manejo de contenidos, } \\
\text { medio y tecnologías. }\end{array}$ & $\begin{array}{l}\text { - Contenidos. } \\
\text { - Medios. } \\
\text { - Tecnología. }\end{array}$ \\
\hline \multirow{4}{*}{ EBIM } & 2 & Almacén de datos de análisis para un negocio específico. & - Data Mart. \\
\hline & 3 & Almacén de datos interactivos, para un análisis más profundo. & - Data Warehouse. \\
\hline & 4 & Integración de máquinas que combinan estructuras analíticas. & $\begin{array}{l}\text { - Enterprise Data } \\
\text { Warehouse. }\end{array}$ \\
\hline & 5 & $\begin{array}{l}\text { Se propagan los cambios de datos a todas las aplicaciones que lo } \\
\text { necesitan. }\end{array}$ & $\begin{array}{l}\text { - Convergencia de datos } \\
\text { empresariales. }\end{array}$ \\
\hline EBI2M & 2 & Gestiona el cambio cultural, organizativo y de las personas. & $\begin{array}{l}\text { - Cambio. } \\
\text { - Administración. }\end{array}$ \\
\hline SOBI & 4 & Proporciona herramientas para integrar aplicaciones. & $\begin{array}{l}\text { - Sistemas orientados a } \\
\text { los servicios. }\end{array}$ \\
\hline \multirow{4}{*}{ TDWI } & 2 & Demandas de información para usuarios de un departamento. & - Spread Marts. \\
\hline & 3 & $\begin{array}{l}\text { La gestión en BI es manejada por un grupo de personas de distintos } \\
\text { departamentos. }\end{array}$ & - Data Marts. \\
\hline & 4 & $\begin{array}{l}\text { Se establecen fuentes de datos centralizadas en BI, con una arquitectura } \\
\text { en común para los Data Warehouse. }\end{array}$ & $\begin{array}{l}\text { - Data Warehouse. } \\
\text { - KPI. }\end{array}$ \\
\hline & 5 & $\begin{array}{l}\text { Se convierten los sistemas de BI en servicios técnicos y de negocios, } \\
\text { buscando la excelencia. }\end{array}$ & $\begin{array}{l}\text { - Enterprise Data } \\
\text { Warehouse. }\end{array}$ \\
\hline
\end{tabular}

Tabla 9. Resultados método MESME.

\begin{tabular}{|l|c|c|c|c|c|}
\hline Modelos & EI & EBI & EBI2 & TDWI & SOBI \\
\hline KPA & & & $\mathrm{X}$ & & \\
\hline Administración & & & $\mathrm{X}$ & & \\
\hline Cambio & $\mathrm{X}$ & & & & \\
\hline $\begin{array}{l}\text { Contenido } \\
\text { datos emprescia de }\end{array}$ & & $\mathrm{X}$ & & & \\
\hline Data Marts & & $\mathrm{X}$ & & $\mathrm{X}$ & \\
\hline Data Warehouse & & $\mathrm{X}$ & & $\mathrm{X}$ & \\
\hline $\begin{array}{l}\text { Enterprise Data } \\
\text { Warehouse }\end{array}$ & & $\mathrm{X}$ & & $\mathrm{X}$ & \\
\hline $\begin{array}{l}\text { Indice clave de } \\
\text { desempeño }\end{array}$ & & & & $\mathrm{X}$ & \\
\hline Medios & $\mathrm{X}$ & & & & \\
\hline $\begin{array}{l}\text { Sistemas orientados } \\
\text { a los servicios }\end{array}$ & & & & & $\mathrm{X}$ \\
\hline Spread Marts & & & & $\mathrm{X}$ & \\
\hline Tecnología & $\mathrm{X}$ & & & & \\
\hline
\end{tabular}

Tabla 10. Resumen resultado método MESME.

\begin{tabular}{|l|c|c|c|c|c|}
\hline Modelos & EI & EBIM & EBI2M & TDWI & SOBI \\
\hline EI & & 0 & 0 & 0 & 0 \\
\hline EBIM & 0 & & 0 & 3 & 0 \\
\hline EBI2M & 0 & 0 & & 0 & 0 \\
\hline TDWI & 0 & 3 & 0 & & 0 \\
\hline SOBI & 0 & 0 & 0 & 0 & \\
\hline
\end{tabular}

En este gráfico se aprecia que solo los modelos de madurez EI y SOBI difieren de la máxima eficiencia alcanzada por los modelos EBIM, TDWI y EBI2M.

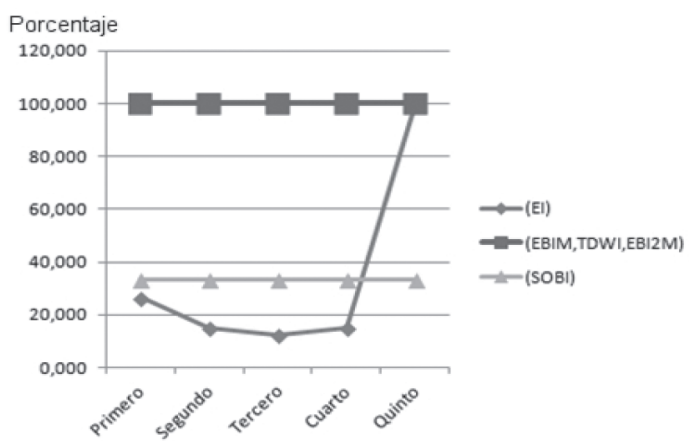

Figura 5. Resumen de eficiencia de todas las DMU.

Es difícil emitir una resolución acerca de las eficiencias relativas de los diferentes modelos de madurez en BI. Esto debido a que la eficiencia de los modelos depende de múltiples factores, entre ellos el enfoque y la estructura, entre otros. Por lo que con la aplicación de la técnica DEA se obtuvo una caracterización de la eficiencia de los niveles para un conjunto de modelos de madurez en BI. 


\section{CONCLUSIONES}

Con la aplicación de la técnica DEA se realizó una caracterización de la capacidad cuantitativa de los diferentes modelos en una determinada fase para transformar las entradas en salidas, en los distintos niveles de madurez para el conjunto de modelos analizados. Para ello se definieron criterios de las entradas y salidas, estos criterios abarcaron la totalidad de los elementos presentes en los distintos modelos analizados. Una vez cuantificadas las entradas y las salidas en cada modelo para todos sus niveles de madurez, se obtuvo el valor de un indicador de la eficiencia entendida como su capacidad cuantitativa para una determinada fase de transformar un cierto número de entradas en un cierto número de salidas. Aunque esta definición de eficiencia es restringida y no general, sí es operacional y mide un aspecto cuantitativo de cada nivel de un modelo de madurez de BI.

Al analizar la eficiencia obtenida en los modelos se aprecia que la mayoría de estos carecen de una caracterización explícita para los elementos que lo componen. Ya que la eficiencia empírica de los modelos EBIM, TDWI y EBI2M es de $100 \%$ en todos sus niveles de madurez, lo que indica que estos modelos poseen el mismo número de entradas y salidas en todos sus niveles de madurez. En el caso del modelo SOBI también presenta una eficiencia constante, pero de $36 \%$ en todos sus niveles de madurez, es decir, el número de entradas y salidas tampoco varía en todos sus niveles de madurez. En cambio, el modelo EI presenta una eficiencia que varía en todos sus niveles de madurez, por ende sus entradas y salidas cambian según las dificultades del nivel de madurez en donde se encuentre.

En el proceso realizado para la aplicación del método MESME se ponderaron todos los elementos que componen un modelo de madurez, con esto se identificó el modelo Enterprise Intelligence como el modelo de referencia para la implementación del método MESME. Posteriormente se definió el objetivo y el alcance del análisis, lo que llevó a la elaboración de un cuestionario para identificar temas comunes presentes en la caracterización del conjunto de modelos, según un propósito y un nivel de detalle específico.

Las escasas similitudes de elementos encontradas en el conjunto de modelos demuestra la diversidad de enfoques que presentan la mayoría de los modelos de madurez en BI analizados. Ya que solo el modelo TDWI posee tres similitudes con el modelo EBI, para los demás modelos no se identificaron similitudes entre ellos.

Con la aplicación de la técnica DEA y el método MESME se infiere que los modelos de madurez en BI existentes poseen diferentes enfoques, entre ellos están la cultura, sistemas o datos, procesos, data warehousing o servicios. La gran mayoría de estos modelos están orientados a una dimensión particular.

La mayoría de los modelos de madurez en BI presentan una escasa caracterización de sus niveles de madurez, ya que no poseen una descripción explícita de los elementos que lo conforman, entre los cuales están las KPA, objetivos específicos y prácticas específicas, entre otros.

El modelo EI es el único que presenta una descripción explícita en todos los elementos que componen un modelo de madurez como son los niveles, KPA, objetivos y prácticas específicas. Este modelo también comprende tres dimensiones esenciales en la arquitectura de Business Intelligence, estas son: proceso, sistemas y datos. Además, el modelo EI es el único que no presenta una estructura rígida, ya que varía en la cantidad de esfuerzos realizados según las dificultades presentadas en el nivel de transición en el que se encuentra la organización.

Debido a que el concepto de Inteligencia Empresarial (Enterprise Intelligence, EI) es más amplio que BI, se puede ocupar como modelo de madurez para BI, obteniendo mayores beneficios, ya que no solo contempla el análisis de los datos, sistemas y procesos, sino también la arquitectura y la gestión del conocimiento.

En este contexto, el modelo EI, por las características que posee, se debería aplicar en una gran organización en Chile que ya tenga implementadas un cierto número mínimo de iniciativas en BI.

El objetivo final de la investigación en desarrollo es la elaboración de un proceso metodológico para guiar la implementación de mejoras en la madurez en BI en una organización. Esta metodología estará enfocada a apoyar la transición de nivel de madurez 
en BI desde el nivel actual al siguiente nivel, para un área de proceso clave (KPA) en particular, esta KPA será seleccionada según la recomendación de expertos. Por lo anterior, el resultado de la presente investigación representa una base para la elección de un modelo de madurez en BI como modelo de referencia para la formulación de la guía metodológica.

\section{REFERENCIAS}

[1] L. Xu, L. Zeng, Z. Shi, Q. He and M. Wang. "Research on Business Intelligence in Enterprise Computing Environment". 2007. DOI: IEEE10.1109/ICSMC.2007.4413870.

[2] B. Wixom and H. Watson. "The Bi-Based organization". University of Virginia and University of Georgia. USA. 2010.

[3] Z. Jourdan, R. Rainer and T. Marshall. "Business intelligence: An analysis of the literature". Information Systems Management. Vol. 25, Issue 2, pp. 121-131. 2008. DOI: $10.1080 / 10580530801941512$.

[4] Gartner Group. "How Secure Is Your Business Intelligence Environment", pp. 1-6. 2002.

[5] I. Hribar Rajterič. "Overview of Business Intelligence Maturity Models". Int. J. Hum. Sci. Vol. 15, Issue 1, pp. 47-67. 2010.

[6] CMMI Product Team. "CMMI for Software Engineering". Version 1.1, Staged. 2002.

[7] W. Cooper, L. Seiford and K. Tone. "Data Envelopment Analysis". Edit. Kluwer Academic Publishers. 2000.

[8] J. Calvo-Manzano. "Process Similarity Study: Case Study on Project Planning Practices Based on CMMI-DEV v1.2". EuroSPI' 2008 Industrial Proceedings. 2008.

[9] M. Chuah and K. Wong. "A review of business intelligence and its maturity models".
University Tunku Abdul Rahman, Malaysia. 2011.

[10] G. Lahrmann, F. Marx, R. Winter and F. Wortmann. "Business Intelligence Maturity Models: An Overview". VII Conference of the Italian Chapter of AIS. Naples, Italy. 2010.

[11] J. Huffman and L. Whitma. "Developing a Capability Maturity Model for Enterprise Intelligence". Preprints of the 18th IFAC World Congress Milano, Italy. 2011.

[12] C. Tanl, Y. Siml and W. Yeoh. "A Maturity Model of Enterprise Business Intelligence". 2011.

[13] E. Shaaban. "Business Intelligence Maturity Models: Toward New Integrated Model". 2011.

[14] W. Eckerson. "Interpreting Benchmark Score Using TDWI's Maturity Model". TDWI Benchmark Guide. 2007. URL: http://onereports.inquisiteasp.com/Docs/ TDWI_Benchmark_Final.pdf. Date of visit: May 16, 2011.

[15] M. Chuah and K. Wong. "Construct an Enterprise Business Intelligence Maturity Model (EBI2M) Using an Integration Approach: A Conceptual Framework". University Tunku Abdul Rahman. Malaysia. 2011.

[16] J. López and S. Henao. "Aplicación de la técnica DEA (Data Envelopment Analysis) en la determinación de eficiencia de centros de costos de producción". Universidad Tecnológica de Pereira. 2007. ISSN: 0122-1701.

[17] D. Wells. "Business Analytics-Getting the Point". 2008. URL: http://b-eye-network. $\mathrm{com} / \mathrm{view} / 7133$. Date of visit: October 11, 2011. 